\title{
Ablation of atrioventricular nodal re-entrant tachycardia is safe and effective in the elderly
}

Cardiovascular interventional therapies are often withheld from elderly patients, owing to a perceived increase in the risk of complications. Catheter ablation for atrioventricular nodal re-entrant tachycardia (AVNRT) is no exception. This procedure has been associated with complete atrioventricular (AV) block in elderly patients, although the data are inconsistent. Boris Hoffmann and colleagues have now reported that ablation can be performed for AVNRT in patients aged $>75$ years with equal safety and efficacy as in younger patients. In their paper, which is published in Heart Rhythm, the investigators concluded that "because antiarrhythmic drug therapy is often ineffective in this age group [ $\geq 75$ years], catheter ablation of AVNRT should be considered the preferred treatment option, even in elderly patients".

\section{4 ...little data exist on the use of catheter ablation for AVNRT in elderly patients... 77}

AVNRT usually affects individuals aged $<30$ years, and is relatively uncommon in those aged $>60$ years. Consequently, little data exist on the outcomes of catheter ablation for AVNRT in elderly patients. This procedure is, however, the treatment of choice for younger patients, and is curative in $90 \%$ of cases. Researchers at the Institute for Research in Myocardial Infarction (Ludwigshafen, Germany) set up the German Ablation Registry to investigate long-term outcomes among patients with AVNRT undergoing catheter ablation, including those aged $\geq 75$ years. The registry included data from 48 centers.

Between March 2007 and May 2010, 3,234 patients were enrolled in the registry. Patients were divided into three groups: $<50$ years of age (group $1 ; n=1,268$ ), $50-75$ years of age (group $2 ; n=1,707$ ), and $>75$ years of age (group $3 ; n=259$ ). Ablation of the slow AV nodal pathway was performed using radiofrequency energy $(97.7 \%)$ or cryotherapy (2.3\%) and monitored using continuous fluoroscopy. The primary efficacy end point was the noninducibility of AVNRT with a maximum of one AV nodal echo beat when using programmed atrial stimulation.

Patients in group 3 had comorbidities (including heart failure, diabetes mellitus, and syncope) more often than those in groups 1 and 2. Compared with the younger age groups, more patients in group 3 were refractory to antiarrhythmic drug therapy. The rate of procedural success (primary end point) was high in all three groups (98.598.8\%), with no significant between-group differences. Similarly, no differences in the total rate of complications were reported between the three groups. Hemodynamically stable pericardial effusion occurred in seven patients (five

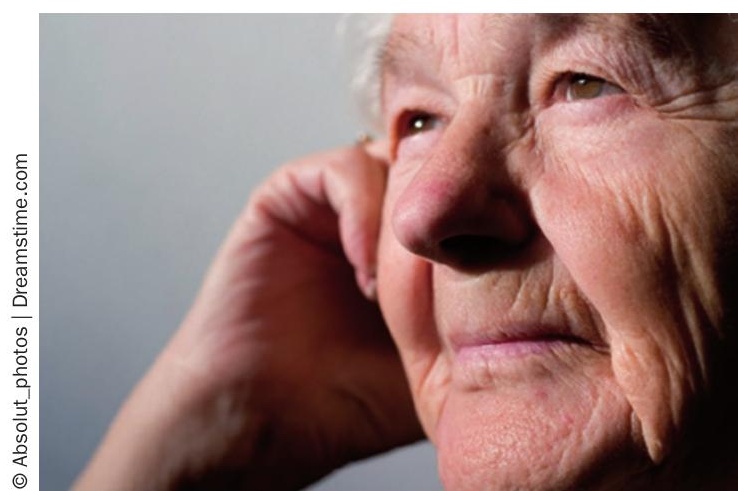

in group 2 and two in group 3), although none required pericardiocentesis. Only six patients experienced AV block; none of these individuals were aged $>75$ years. For patients in group 3, the fluoroscopy dose was higher, the duration of fluoroscopy was longer, and the radiofrequency energy lower than in the other two groups. These finding lead Hoffman et al. to speculate that ablation might have been performed more carefully in elderly patients than in younger individuals and that "due to degenerative changes in the AV node of older patients, less energy is necessary to reach the end point".

\section{Alexandra King}

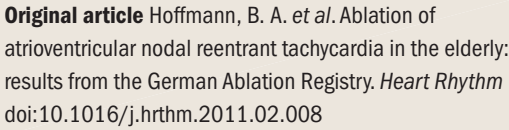

